A unified framework for the evaluation of surrogate endpoints in mental-health clinical trials

Peer-reviewed author version

MOLENBERGHS, Geert; BURZYKOWSKI, Tomasz; ALONSO ABAD, Ariel; ASSAM NKOUIBERT, Pryseley; Tilahun, Abel \& BUYSE, Marc (2010) A unified framework for the evaluation of surrogate endpoints in mental-health clinical trials. In:

STATISTICAL METHODS IN MEDICAL RESEARCH, 19 (3) p. 205-236.

DOI: $10.1177 / 0962280209105015$

Handle: http://hdl.handle.net/1942/11049 


\title{
A Unified Framework for the Evaluation of Surrogate Endpoints in Mental-Health Clinical Trials
}

\author{
Geert Molenberghs $^{1,2} \quad$ Tomasz Burzykowski $^{1} \quad$ Ariel Alonso $^{1}$ \\ Pryseley Assam $^{1} \quad$ Abel Tilahun $^{1}$ \\ Marc Buyse $e^{3,1}$ \\ ${ }^{1}$ I-BioStat, Hasselt University, Diepenbeek, Belgium \\ 2 I-BioStat, Katholieke Universiteit Leuven, Leuven Belgium \\ ${ }^{3}$ International Drug Development Institute, Ottignies Louvain-la-Neuve, Belgium
}

\begin{abstract}
For a number of reasons, surrogate endpoints are considered instead of the so-called true endpoint in clinical studies, especially when such endpoints can be measured earlier, and/or with less burden for patient and experimenter. Surrogate endpoints may occur more frequently than their standard counterparts. For these reasons, it is not surprising that the use of surrogate endpoints in clinical practice is increasing.

Building on the seminal work of Prentice (1) and Freedman, Graubard, and Schatzkin(2), Buyse et al(3) framed the evaluation exercise within a meta-analytic setting, in an effort to overcome difficulties that necessarily surround evaluation efforts based on a single trial. In this paper, we review the meta-analytic approach for continuous outcomes, discuss extensions to non-normal and longitudinal settings, as well as proposals to unify the somewhat disparate collection of validation measures currently on the market. Implications for design and for predicting the effect of treatment in a new trial, based on the surrogate, are discussed. A case study in schizophrenia is analyzed.
\end{abstract}

Some Key Words: Hierarchical model; Information theory; Likelihood reduction factor; Metaanalysis; Random-effects model; Surrogate endpoint; Surrogate threshold effect.

\section{Introduction}

The rising costs of drug development and the challenges of new and re-emerging diseases are putting considerable demands on efficiency in the drug candidates selection process. A very important factor influencing duration and complexity of this process is the choice of endpoint used to assess drug efficacy. Often, the most sensitive and relevant clinical endpoint might be difficult to use in a trial. This happens if measurement of the clinical endpoint (1) is costly (e.g., to diagnose cachexia, a condition associated with malnutrition and involving loss of muscle and fat tissue, expensive equipment measuring content of 
nitrogen, potassium and water in patients' body is required); (2) is difficult (e.g., involving compound measures such as encountered in quality-of-life or pain assessment); (3) requires a long follow-up time (e.g., survival in early stage cancers); or (4) requires a large sample size because of low event incidence (e.g., short-term mortality in patients with suspected acute myocardial infarction). An effective strategy is then proper selection and application of biomarkers for efficacy, replacing the clinical endpoint by a biomarker that is measured more cheaply, more conveniently, more frequently, or earlier. From a regulatory perspective, a biomarker is considered acceptable for efficacy determination only after its establishment as a valid indicator of clinical benefit, i.e., after its validation as a surrogate marker(4).

These considerations naturally lead to the need of proper definitions. An important step came from the Biomarker Definitions Working Group $(6 ; 5)$, their definitions nowadays being widely accepted and adopted. A clinical endpoint is considered the most credible indicator of drug response and defined as a characteristic or variable that reflects how a patient feels, functions, or survives. During clinical trials, endpoints should be used, unless a biomarker is available that has risen to the status of surrogate endpoint. A biomarker is defined as a characteristic that can be objectively measured as an indicator of healthy or pathological biological processes, or pharmacological responses to therapeutic intervention. A surrogate endpoint is a biomarker, intended for substituting a clinical endpoint. A surrogate endpoint is expected to predict clinical benefit, harm, or lack of these.

Surrogate endpoints have been used in medical research for a long time(7; 8). Owing to unfortunate historical events and in spite of potential advantages, their use has been surrounded by controversy. The best known case is the approval by the Food and Drug Administration (FDA) of three antiarrhythmic drugs: encainide, flecainide, and moricizine. The drugs were approved because of their capacity to effectively suppress arrhythmias. It was believed that, because arrhythmia is associated with an almost fourfold increase in the rate of cardiac-complication-related death, the drugs would reduce the death rate. However, a post-marketing trial showed that the active-treatment death rate was double the placebo rate. A risk was also detected for moricizine(9). Another example came with the surge of the AIDS epidemic. The impressive early therapeutic results obtained with zidovudine, and the pressure for accelerated evaluation of new therapies, led to the use of CD4 blood count as a surrogate endpoint for time to clinical events and overall survival(10), in spite of concern about its limitations as a surrogate marker for clinically relevant endpoints(11). 
The main reason behind failures was the incorrect perception that surrogacy simply follows from the association between a potential surrogate endpoint and the corresponding clinical endpoint, the mere existence of which is insufficient for surrogacy(8). Even though the existence of an association between the potential surrogate and the clinical endpoint is undoubtedly a desirable property, what is required to replace the clinical endpoint by the surrogate is that the effect of the treatment on the surrogate endpoint reliably predicts the effect on the clinical endpoint. Partly owing to the lack of appropriate methodology, this condition was not checked in the early attempts and, consequently, negative opinions about the use of surrogates in the evaluation of treatment efficacy emerged $(8 ; 12 ; 13)$.

Currently, the steady advance in many medical and biological fields is dramatically increasing the number of biomarkers and hence potential surrogate endpoints. Additionally, an increasing number of new drugs have well-defined mechanisms of action at molecular level, allowing drug developers to measure the effect of these drugs on the relevant biomarkers(14). There is also increasing public pressure for fast approval of promising drugs, which will have to be based on biomarkers rather than on long-term, costly clinical endpoints(15). Obviously, the pressure will be especially high when a rapidly increasing incidence of the targeted disease could become a serious threat to public health or the patient's (quality of) life. Shortening the duration of clinical trials not only can decrease the cost of the evaluation process but also limit potential problems with noncompliance and missing data, which are more likely in longer studies $(4 ; 16)$.

Surrogate endpoints can play a role in the earlier detection of safety signals that could point to toxic problems with new drugs. The duration and sample size of clinical trials aimed at evaluating the therapeutic efficacy of new drugs are often insufficient to detect rare or late adverse effects $(17 ; 18)$; using surrogate endpoints in this context might allow one to obtain information about such effects even during the clinical testing phase. Discoveries in medicine and biology are further creating a exciting range of possibilities for the development of potentially effective treatments. This is an achievement, but it also faces us with the challenge of coping with a large number of new promising treatments that should be rapidly evaluated. This is already clear in oncology, because the increased knowledge about the genetic mechanisms operating in cancer cells led to the proposing of novel cancer therapies, such as the use of a genetically-modified virus that selectively attacks p53-deficient cells, sparing normal cells(1). Validated surrogate endpoints can offer an efficient route. The role of surrogate endpoints may 
depend on the trials phase. Nowadays, their use is more accepted in early phases of clinical research, such as in phase II or early phase III clinical trials. Using them to substitute for the clinical endpoint in pivotal phase III trials or to replace the clinical endpoint altogether in all clinical research past a certain point is, however, a topic of ongoing debate. It is difficult to precisely define the future role of surrogate endpoint in the various trial phases. Ultimately, the combination of medical and statistical elements, together with practical and economical considerations, will help answer this question. While the huge potential of surrogate endpoints to accelerate and improve the quality of clinical trials is unquestioned, the above considerations indicate that only thoroughly evaluated surrogates should be used.

It is thus best to use validated surrogates, though one needs to reflect on the precise meaning and extent of validation(19). Like in many clinical decisions, statistical arguments will play a major role, but ought to be considered in conjunction with clinical and biological evidence. At the same time, surrogate endpoints can play different roles in different phases of drug development. While it may be more acceptable to use surrogates in early phases of research, there should be much more restraint in using them as substitutes for the true endpoint in pivotal phase III trials, since the latter might imply replacing the true endpoint by a surrogate for all future studies as well, a far-reaching decision. For a biomarker to be used as a "valid" surrogate, a number of conditions must be fulfilled. The ICH Guidelines on Statistical Principles for Clinical Trials state that "In practice, the strength of the evidence for surrogacy depends upon (i) the biological plausibility of the relationship, (ii) the demonstration in epidemiological studies of the prognostic value of the surrogate for the clinical outcome and (iii) evidence from clinical trials that treatment effects on the surrogate correspond to effects on the clinical outcome" (20)

A motivating case study is introduced in Section 2. A perspective on data from a single trial is given in Section 3. The meta-analytic evaluation framework is presented in Section 4, in the context of normally distributed outcomes. Extensions to a variety of non-Gaussian settings are discussed in Section 5. Efforts for unifying the scattered suite of validation measures are reviewed in Section 6. A number of alternative computational techniques and validation paradigms is presented in Section 7. Implications for prediction of the effect in a new trial and for designing studies based on surrogates are the topics of Section 8. 


\section{A Meta-analysis of Five Clinical Trials in Schizophrenia}

The data come from a meta-analysis of five double-blind randomized clinical trials, comparing the effects of risperidone to conventional anti psychotic agents for the treatment of chronic schizophrenia. The treatment indicator for risperidone versus conventional treatment will be denoted by $Z$. Schizophrenia has long been recognized as a heterogeneous disorder with patients suffering from both 'negative' and 'positive' symptoms. Negative symptoms are characterized by deficits in cognitive, affective and social functions, for example poverty of speech, apathy and emotional withdrawal. Positive symptoms entail more florid symptoms such as delusions, hallucinations and disorganized thinking, which are superimposed on mental status(21). Several measures can be considered to asses a patient's global condition. Clinician's Global Impression (CGI) is generally accepted as a clinical measure of change, even though it is somewhat subjective. Here, the change of CGI versus baseline will be considered as the true endpoint $T$. It is scored on a 7-grade scale used by the treating physician to characterize how well a subject has improved since baseline. Another useful and sufficiently sensitive assessment scales is the Positive and Negative Syndrome Scale (PANSS)(22). The PANSS consists of 30 items that provide an operationalized, drug-sensitive instrument, which is highly useful for both typological and dimensional assessment of schizophrenia. We will use the change versus baseline in PANSS as our surrogate $S$. The data contain five trials and in all trials, information is available on the investigators that treated the patients. This information is helpful to define group of patients that will become units of analysis. Figure 1 displays the individual profiles (some of them have been highlighted) for each scale by treatment group. It seems that, on average, these profiles follow a linear trend over time and the variability seems to be constant over time.

\section{Data from a Single Unit}

In this section, we will discuss the single unit setting (e.g., a single trial). The notation and modeling concepts introduced are useful to present and critically discuss the key ingredients of the PrenticeFreedman framework. Therefore, this section should not be seen as setting the scene for the rest of the paper. This is reserved for the multi-unit case (Section 4). Criticisms towards this framework can also be found in Joffe and Greene(23).

Throughout the paper, we will adopt the following notation: $T$ and $S$ are random variables that denote 
the true and surrogate endpoints, respectively, and $Z$ is an indicator variable for treatment. For ease of exposition, we will assume that $S$ and $T$ are normally distributed. The effect of treatment on $S$ and $T$ can be modeled as follows:

$$
\begin{aligned}
& S_{j}=\mu_{S}+\alpha Z_{j}+\varepsilon_{S j}, \\
& T_{j}=\mu_{T}+\beta Z_{j}+\varepsilon_{T j},
\end{aligned}
$$

where $j=1, \ldots, n$ indicates patients, and the error terms have a joint zero-mean normal distribution with covariance matrix

$$
\Sigma=\left(\begin{array}{cc}
\sigma_{S S} & \sigma_{S T} \\
& \sigma_{T T}
\end{array}\right) .
$$

In addition, the relationship between $S$ and $T$ can be described by a regression of the form

$$
T_{j}=\mu+\gamma S_{j}+\varepsilon_{j}
$$

Note that this model is introduced because it is a component of the Prentice-Freedman framework. Given that the fourth criterion will involve a dependence on the treatment as well, as in (5), it is of legitimate concern to doubt whether (4) and (5) are simultaneously plausible. Also, the introduction of (4) should not be seen as an implicit of explicit assumption about the absence of treatment effect in the regression relationship, but rather as a model that can be used, when the uncorrected association between both endpoints is of interest.

We will assume later (Section 4) that the $n$ patients come from $N$ different experimental units, but for now the simple situation of a single experiment will suffice to explore some fundamental difficulties with the validation of surrogate endpoints.

\subsection{Definition and Criteria}

Prentice(1) proposed to define a surrogate endpoint as "a response variable for which a test of the null hypothesis of no relationship to the treatment groups under comparison is also a valid test of the corresponding null hypothesis based on the true endpoint" ((1) p. 432). In terms of our simple model (1)-(2), the definition states that for $S$ to be a valid surrogate for $T$, parameters $\alpha$ and $\beta$ must simultaneously be equal to, or different from, zero. This definition is not consistent with the availability of a single experiment only, since it requires a large number of experiments to be available, each with 
tests of hypothesis on both the surrogate and true endpoints. An important drawback is also that evidence from trials with non-significant treatment effects cannot be used, even though such trials may be consistent with a desirable relationship between both endpoints. Prentice derived operational criteria that are equivalent to his definition. These criteria require that

- treatment has a significant impact on the surrogate endpoint (parameter $\alpha$ differs significantly from zero in $(1))$,

- treatment has a significant impact on the true endpoint (parameter $\beta$ differs significantly from zero in (2)),

- the surrogate endpoint has a significant impact on the true endpoint (parameter $\gamma$ differs significantly form zero in (4)), and

- the full effect of treatment upon the true endpoint is captured by the surrogate.

The last criterion is verified through the conditional distribution of the true endpoint, given treatment and surrogate endpoint, derived from (1)-(2):

$$
T_{j}=\tilde{\mu}_{T}+\beta_{S} Z_{j}+\gamma_{Z} S_{j}+\tilde{\varepsilon}_{T j}
$$

where the treatment effect (corrected for the surrogate $S$ ), $\beta_{S}$, and the surrogate effect (corrected for treatment $Z), \gamma_{Z}$, are

$$
\begin{aligned}
& \beta_{S}=\beta-\sigma_{T S} \sigma_{S S}^{-1} \alpha, \\
& \gamma_{z}=\sigma_{T S} \sigma_{S S}^{-1},
\end{aligned}
$$

and the variance of $\tilde{\varepsilon}_{T j}$ is given by

$$
\sigma_{T T}-\sigma_{T S}^{2} \sigma_{S S}^{-1}
$$

It is usually stated that the fourth criterion requires that the parameter $\beta_{S}$ be equal to zero (we return to this notion in Section 3.3). Essentially, this last criterion states that the true endpoint $T$ is completely determined by knowledge of the surrogate endpoint $S$. Buyse and Molenberghs(24) showed that the last two criteria are necessary and sufficient for binary responses, but not in general. Several authors, including Prentice, pointed out that the criteria are too stringent to be fulfilled in real situations $(1)$. 
In spite of these criticisms, the spirit of the fourth criterion is very appealing. This is especially true if it can be considered in the light of an underlying biological mechanism. For example, it is interesting to explore whether the surrogate is part of the causal chain leading from treatment exposure to the final endpoint. While this issue is beyond the scope of the current paper, the connection between statistical validation (with emphasis on association) and biological relevance (with emphasis on causation) deserves further reflection.

\subsection{The Proportion Explained}

Freedman, Graubard, and Schatzkin(2) argued that the last Prentice criterion raises a conceptual difficulty since it requires the statistical test for treatment effect on the true endpoint to be non-significant after adjustment for the surrogate. The non-significance of this test does not prove that the effect of treatment upon the true endpoint is fully captured by the surrogate, and therefore Freedman, Graubard, and Schatzkin(2) proposed to calculate the proportion of the treatment effect mediated by the surrogate:

$$
P E=\frac{\beta-\beta_{S}}{\beta},
$$

with $\beta_{S}$ and $\beta$ obtained respectively from (5) and (2). In this paradigm, a valid surrogate would be one for which the proportion explained $(P E)$ is equal to one. In practice, a surrogate would be deemed acceptable if the lower limit of its confidence interval of $P E$ was "sufficiently" large.

Some difficulties surrounding the $P E$ have been described in the literature $(24 ; 25 ; 26 ; 27 ; 28 ; 29)$. The $P E$ will tend to be unstable when $\beta$ is close to zero, a situation that is likely to occur in practice. As Freedman, Graubard, and Schatzkin(2) themselves acknowledged, the confidence limits of $P E$ will tend to be rather wide (and sometimes even unbounded if Fieller confidence intervals are used), unless large sample sizes are available or a very strong effect of treatment on the true endpoint is observed. Note that large sample sizes are typically available in epidemiologic studies or in meta-analyses of clinical trials. Another complication arises when (5) is not the correct conditional model, and an interaction term between $Z_{i}$ and $S_{i}$ needs to be included. In that case, defining the $P E$ becomes problematic.

\subsection{The Relative Effect}

Buyse and Molenberghs(24) suggested to calculate another quantity for the validation of a surrogate endpoint: the relative effect $(R E)$, which is the ratio of the effects of treatment upon the final and the 
surrogate endpoint. Formally:

$$
R E=\frac{\beta}{\alpha}
$$

They also considered the treatment-adjusted association between the surrogate and the true endpoint, $\rho_{Z}:$

$$
\rho_{Z}=\frac{\sigma_{S T}}{\sqrt{\sigma_{S S} \sigma_{T T}}} .
$$

Now, a simple relationship can be derived between $P E, R E$, and $\rho_{Z}$. Let us define $\lambda^{2}=\sigma_{T T} \sigma_{S S}^{-1}$. It follows that $\lambda \rho_{Z}=\sigma_{S T} \sigma_{S S}^{-1}$ and, from (6), $\beta_{S}=\beta-\rho_{Z} \lambda \alpha$. As a result, we obtain

$$
P E=\lambda \rho_{Z} \frac{\alpha}{\beta}=\lambda \rho_{Z} \frac{1}{R E}
$$

A similar relationship was derived by Buyse and Molenberghs(24) and by Begg and Leung(30) for standardized surrogate and true endpoints. Let us now turn to the more promising meta-analytic framework.

\section{A Meta-analytic Framework for Normally Distributed Outcomes}

Several methods have been suggested for the formal evaluation of surrogate markers, some based on a single trial with others, currently gaining momentum, of a meta-analytic nature. The first formal single trial approach to validate markers is due to Prentice(1), who gave a definition of the concept of a surrogate endpoint, followed by a series of operational criteria. Freedman, Graubard, and Schatzkin(2) augmented Prentice's hypothesis-testing based approach, with the estimation paradigm, through the so-called proportion of treatment effect explained. In turn, Buyse and Molenberghs(24) added two further measures: the relative effect and the adjusted association. All of these proposals are hampered by the fact that they are single-trial based, in which there evidently is replication at the patient level, but not at the level of the trial.

\subsection{A Meta-Analytic Approach}

Although the single trial based methods are relatively easy in terms of implementation, they are surrounded with the difficulties stated at the end of the previous section. Therefore, several authors, such as Daniels and Hughes(25), Buyse et al(3), and Gail et al(31) have introduced the meta-analytic ap- 
proach. This section briefly outlines the methodology, followed by simplified modeling approaches as suggested by Tibaldi et al(32).

The meta-analytic approach was formulated originally for two continuous, normally distributed outcomes, and extended in the meantime to a large collection of outcome types, ranging from continuous, binary, ordinal, time-to-event, and longitudinally measured outcomes(4). First, we focus on the continuous case, where the surrogate and true endpoints are jointly normally distributed.

The method is based on a hierarchical two-level model. Both a fixed-effects and a random-effects view can be taken. Let $T_{i j}$ and $S_{i j}$ be the random variables denoting the true and surrogate endpoints for the $j$ th subject in the $i$ th trial, respectively, and let $Z_{i j}$ be the indicator variable for treatment. First, consider the following fixed-effects models:

$$
\begin{aligned}
& S_{i j}=\mu_{S i}+\alpha_{i} Z_{i j}+\varepsilon_{S i j}, \\
& T_{i j}=\mu_{T i}+\beta_{i} Z_{i j}+\varepsilon_{T i j},
\end{aligned}
$$

where $\mu_{S i}$ and $\mu_{T i}$ are trial-specific intercepts, $\alpha_{i}$ and $\beta_{i}$ are trial-specific effects of treatment $Z_{i j}$ on the endpoints in trial $i$, and $\varepsilon_{S i}$ and $\varepsilon_{T i}$ are correlated error terms, assumed to be zero-mean normally distributed with covariance matrix

$$
\boldsymbol{\Sigma}=\left(\begin{array}{cc}
\sigma_{S S} & \sigma_{S T} \\
& \sigma_{T T}
\end{array}\right) .
$$

In addition, we can decompose

$$
\left(\begin{array}{c}
\mu_{S i} \\
\mu_{T i} \\
\alpha_{i} \\
\beta_{i}
\end{array}\right)=\left(\begin{array}{c}
\mu_{S} \\
\mu_{T} \\
\alpha \\
\beta
\end{array}\right)+\left(\begin{array}{c}
m_{S i} \\
m_{T i} \\
a_{i} \\
b_{i}
\end{array}\right),
$$

where the second term on the right hand side of (15) is assumed to follow a zero-mean normal distribution with covariance matrix

$$
D=\left(\begin{array}{cccc}
d_{S S} & d_{S T} & d_{S a} & d_{S b} \\
& d_{T T} & d_{T a} & d_{T b} \\
& & d_{a a} & d_{a b} \\
& & & d_{b b}
\end{array}\right) .
$$

A classical hierarchical, random-effects modeling strategy results from the combination of the above two steps into a single one:

$$
S_{i j}=\mu_{S}+m_{S i}+\alpha Z_{i j}+a_{i} Z_{i j}+\varepsilon_{S i j},
$$




$$
T_{i j}=\mu_{T}+m_{T i}+\beta Z_{i j}+b_{i} Z_{i j}+\varepsilon_{T i j}
$$

Here, $\mu_{S}$ and $\mu_{T}$ are fixed intercepts, $\alpha$ and $\beta$ are fixed treatment effects, $m_{S i}$ and $m_{T i}$ are random intercepts, and $a_{i}$ and $b_{i}$ are random treatment effects in trial $i$ for the surrogate and true endpoints, respectively. The random effects $\left(m_{S i}, m_{T i}, a_{i}, b_{i}\right)$ are assumed to be mean-zero normally distributed with covariance matrix (16). The error terms $\varepsilon_{S i j}$ and $\varepsilon_{T i j}$ follow the same assumptions as in the fixed effects models.

After fitting the above models, surrogacy is captured by means of two quantities: trial-level and individual-level coefficients of determination. The former quantifies the association between the treatment effects on the true and surrogate endpoints at the trial level, while the latter measures the association at the level of the individual patient, after adjustment for the treatment effect. The former is given by:

$$
R_{\text {trial }}^{2}=R_{b_{i} \mid m_{S i}, a_{i}}^{2}=\frac{\left(\begin{array}{c}
d_{S b} \\
d_{a b}
\end{array}\right)^{T}\left(\begin{array}{ll}
d_{S S} & d_{S a} \\
d_{S a} & d_{a a}
\end{array}\right)^{-1}\left(\begin{array}{l}
d_{S b} \\
d_{a b}
\end{array}\right)}{d_{b b}} .
$$

The above quantity is unitless and, at the condition that the corresponding variance-covariance matrix is positive definite, lies within the unit interval.

Apart from estimating the strength of surrogacy, the above model can also be used for prediction purposes. To this end, observe that $\left(\beta+b_{0} \mid m_{S 0}, a_{0}\right)$ follows a normal distribution with mean and variance:

$$
\begin{aligned}
E\left(\beta+b_{0} \mid m_{S 0}, a_{0}\right) & =\beta+\left(\begin{array}{c}
d_{S b} \\
d_{a b}
\end{array}\right)^{T}\left(\begin{array}{ll}
d_{S S} & d_{S a} \\
d_{S a} & d_{a a}
\end{array}\right)^{-1}\left(\begin{array}{c}
\mu_{S 0}-\mu_{S} \\
\alpha_{0}-\alpha
\end{array}\right), \\
\operatorname{Var}\left(\beta+b_{0} \mid m_{S 0}, a_{0}\right) & =d_{b b}-\left(\begin{array}{c}
d_{S b} \\
d_{a b}
\end{array}\right)^{T}\left(\begin{array}{ll}
d_{S S} & d_{S a} \\
d_{S a} & d_{a a}
\end{array}\right)^{-1}\left(\begin{array}{c}
d_{S b} \\
d_{a b}
\end{array}\right) .
\end{aligned}
$$

A prediction can be made using (20), with prediction variance (21). Of course, one has to properly acknowledge the uncertainty resulting from the fact that parameters are not known but merely estimated. We return to this issue in Section 8.

Models (12) and (13) are referred to as the full fixed-effects models. It is sometimes necessary, for computational reasons, to contemplate a simplified version. A reduced version of these models is obtained by replacing the fixed trial-specific intercepts by a common one. Thus, the reduced mixed 
effect models result from removing the random trial-specific intercepts $m_{S i}$ and $m_{T i}$ from models (17) and (18). The $R^{2}$ for the reduced models then is:

$$
R_{\text {trial(r) }}^{2}=R_{b_{i} \mid a_{i}}^{2}=\frac{d_{a b}^{2}}{d_{a a} d_{b b}} .
$$

A surrogate could be adopted when $R_{\text {trial }}^{2}$ is sufficiently large. Arguably, rather than using a fixed cutoff above which a surrogate would be adopted, there always will be clinical and other judgment involved in the decision process. The $R_{\text {indiv }}^{2}$ is based on (14) and takes the following form:

$$
R_{\text {indiv }}^{2}=R_{\varepsilon_{T i} \mid \varepsilon_{S i}}^{2}=\frac{\sigma_{S T}^{2}}{\sigma_{S S} \sigma_{T T}}
$$

\subsection{Simplified Modeling Strategies}

Though the above hierarchical modeling is elegant, it often poses a considerable computational challenge(4). To address this problem, Tibaldi et al (32) suggested several simplifications, briefly outlined here. These authors considered three possible dimensions along which simplifications can be undertaken.

The first choice is between treating the trial-specific effects as fixed or random. If the trial-specific effects are chosen to be fixed, a two-stage approach is adopted. The first-stage model will take the form (12)-(13) and at the second stage, the estimated treatment effect on the true endpoint is regressed on the treatment effect on the surrogate and the intercept associated with the surrogate endpoint as

$$
\widehat{\beta}_{i}=\widehat{\lambda}_{0}+\widehat{\lambda}_{1} \widehat{\mu}_{S i}+\widehat{\lambda}_{2} \widehat{\alpha}_{i}+\varepsilon_{i}
$$

The trial-level $R_{\text {trial(f) }}^{2}$ then is obtained by regressing $\widehat{\beta}_{i}$ on $\widehat{\mu}_{S i}$ and $\widehat{\alpha}_{i}$, whereas $R_{\text {trial(r) }}^{2}$ is obtained from regressing $\widehat{\beta}_{i}$ on $\widehat{\alpha}_{i}$ only. The individual-level value is calculated as in (22), using the estimates from (14).

The second option is to consider the trial-specific effects as random. Depending on whether the endpoints are considered jointly or separately (see next paragraph), two directions can be followed. The first one involves a two-stage approach with at the first stage univariate models (17)-(18). A second stage model consists of a normal regression with the random treatment effect on the true endpoint as response and the random intercept and random treatment effect on the surrogate as covariates. The second direction is based on a full random effects model. 
Though natural to assume the two endpoints correlated, this can lead to computational difficulties in fitting the models. The need for the bivariate nature of the outcome is associated with $R_{\text {indiv }}^{2}$ which is in some cases of secondary importance. In addition, there is also a possibility to estimate it by making use of the correlation between the residuals from two separate univariate models. Thus, further simplification can be achieved by fitting separate models for the true and surrogate endpoints, the so-called univariate approach.

If in the trial dimension, the trial-specific effects are considered fixed, models (12)-(13) are fitted separately. Similarly, if the trial-specific effects are considered random, models (17)-(18) are fitted separately, i.e., the corresponding error terms in the two models are assumed independent.

When the univariate approach and/or the fixed-effects approach are chosen, there is a need to adjust for the heterogeneity in information content between trial-specific contributions. One way of doing so is weighting the contributions according to trial size. This gives rise to a weighted linear regression model (23) in the second stage.

In summary, the simplified strategies perform rather well, especially when outcomes are of a continuous nature(33), and are a valuable addition to the fully specified hierarchical model, for those situations where the latter is infeasible or less reliable.

\subsection{Some Reflections}

A cornerstone of the meta-analytic method is the choice of unit of analysis such as, for example, trial, center, or investigator. This choice may depend on practical considerations, such as the information available in the data, experts' considerations about the most suitable unit for a specific problem, the amount of replication at a potential unit's level, and the number of patients per unit. From a technical point of view, the most desirable situation is where the number of units and the number of patients per unit is sufficiently large. This issue has been discussed by Cortiñas et al(33). Of course, in cases where one has to resort to simplified strategies, one has to reflect carefully on the status of the results obtained. Arguably, they may not be as reliable as one might hope for, and one should undertake every effort possible to increase the amount of information available. Clearly, even an analysis based on a simplified strategy, especially in the light of good performance, may support efforts to make more data 
available for analysis.

Most of the work reported in Burzykowski, Molenberghs, and Buyse(4) is for a dichotomous treatment indicator. Two choices need to be made at analysis time. First, the treatment variable can be considered continuous or discrete (a class variable). Second, when a continuous route is chosen, it is relevant to reflect on the actual coding, $0 / 1$ and $-1 /+1$ being the most commonly encountered ones. For models with treatment occurring as a fixed effect only, these choices are essentially irrelevant, since all choices lead to an equivalent model fit, with parameters connected by simple linear transformations. Note that this is not the case, of course, for more than three treatment arms. However, of more importance for us here is the impact the choices can have on the hierarchical model. Indeed, while the marginal model resulting from (17)-(18) is invariant under such choices, this is not true for the hierarchical aspects of the model, such as, for example, the $R^{2}$ measures derived at the trial level. Indeed, $a-1 /+1$ coding ensures the same components of variability operate in both arms, whereas a $0 / 1$ coding, for a positive-definite $D$ matrix, forces the variability in the experimental arm to be greater than or equal to the variability in the standard arm. Both situations may be relevant, and it is of importance to illicit views from the study's investigators.

When the full bivariate random effect is used, the $R_{\text {trial }}^{2}$ is computed from the variance-covariance matrix (16). It is sometimes possible that this matrix be ill-conditioned and/or non-positive definite. In such cases, the resulting quantities computed based on this matrix might not be trustworthy. One way to assess the ill-conditioning of a matrix is by reporting its condition number, i.e., the ratio of the largest over the smallest eigenvalue. A large condition number is an indication of ill-conditioning. The most pathological situation occurs when at least one eigenvalue is equal to zero. This corresponds to a positive semi-definite matrix, which occurs, for example, when a boundary solution is obtained. While it is hard to definitively identify the reason for a zero eigenvalue, insufficient information, either in terms of the number of trials, the simple size within trials, or both, may often be the cause and deserving of careful assessment. Using the simplified methods is certainly an option in this case; apart from providing a solution to the problem, it may give a handle on the problem at hand. 


\subsection{Analysis of the Meta-analysis of Five Clinical Trials in Schizophernia}

Let us analyze the schizophrenia study. Here, trial seems the natural unit of analysis. Unfortunately, the number of trials is not sufficient to apply the full meta-analytic approach. The use of trial as unit of analysis for the simplified methods might also entail problems. The second stage involves a regression model based on only five points, which might give overly optimistic or at least unreliable $R^{2}$ values. The other possible unit of analysis for this study is 'investigator'. There were 176 investigators, each treating between 2 and 60 patients. The use of investigator as unit of analysis is also surrounded with problems. Although a large number of investigators is convenient to explain the between investigator variability, because some investigators treated few patients, the resulting within-unit variability might not be estimated correctly.

The basic meta-analytic approach and the corresponding simplified strategies have been applied, with results displayed in Table 1. Investigator and trial were both used as units of analysis. However, as there were only five trials, it became difficult to base the analysis on trial as unit of analysis in the case of the full bivariate random-effects approach. The results have shown a remarkable difference in the two cases. Consistently, in all of the different simplifications, the $R_{\text {trial }}^{2}$ values were found to be higher when trial was used as unit of analysis. The bivariate full random effect model does not converge when trial is used as the unit of analysis. This might be due to lack of sufficient information to compute all sources of variability. The reduced bivariate random effects model converged for both cases, but the resulting variance-covariance matrices were not positive-definite and were ill-conditioned, as can be seen from the very large value of the condition number. Consequently, the results of the bivariate random effects model should be treated with caution. If we concentrate on the results based on investigator as unit of analysis, we observe a low level of surrogacy of PANSS for CGI, with $R_{\text {trial }}^{2}$ ranging roughly between 0.5 and 0.68 for the different simplified models. This result, however, has to be coupled with other findings based on expert opinion to fully guarantee the validation of PANSS as possible surrogate for CGI. Turning to $R_{\text {indiv, }}^{2}$ it ranges between 0.4904 and 0.5230 , depending on the method of analysis, which is relatively low. To conclude, based on the investigators as unit of analysis, PANSS does not seem a promising surrogate for $\mathrm{CGI}$. 


\section{Non-Gaussian Endpoints}

Statistically speaking, the surrogate endpoint and the clinical endpoint are realizations of random variables. As will be clear from the formalism in Section 4, one is in need of the joint distribution of these variables. The easiest, but not the only, situation is where both are Gaussian random variables, but one also encounters binary (e.g., CD4+ counts over 500/mm3, tumor shrinkage), categorical (e.g., cholesterol levels $<200 \mathrm{mg} / \mathrm{dl}, 200-299 \mathrm{mg} / \mathrm{dl}, 300+\mathrm{mg} / \mathrm{dl}$, tumor response as complete response, partial response, stable disease, progressive disease), censored continuous (e.g., time to undetectable viral load, time to cardiovascular death), longitudinal (e.g., CD4+ counts over time, blood pressure over time), and multivariate longitudinal (e.g., CD4+ and viral load over time jointly, various dimensions of quality of life over time) endpoints. The models used to validate a surrogate for a clinical endpoint will depend on the type of variables observed in the problem at hand. Table 2 shows some examples of potential surrogate endpoints in various diseases. In what follows, we will briefly discuss the settings of binary endpoints, failure-time endpoints, the combination of an ordinal and a survival endpoint, and longitudinal endpoints.

\subsection{Binary Endpoints}

Renard et al(34) have shown that extension to this situation is easily done using a latent variable formulation. That is, one posits the existence of a pair of continuously distributed latent variable responses $\left(\widetilde{S}_{i j}, \widetilde{T}_{i j}\right)$ that produce the actual values of $\left(S_{i j}, T_{i j}\right)$. These unobserved variables are assumed to have a joint normal distribution and the realized values follow by double dichotomization. On the latent-variable scale, we obtain a model similar to (12)-(13) and in the matrix (14) the variances are set equal to unity in order to ensure identifiability. This leads to the following model:

$$
\left\{\begin{array}{l}
\Phi^{-1}\left(P\left[S_{i j}=1 \mid Z_{i j}, m_{S_{i}}, a_{i}, m_{T_{i}}, b_{i}\right]\right)=\mu_{S}+m_{S_{i}}+\left(\alpha+a_{i}\right) Z_{i j}, \\
\Phi^{-1}\left(P\left[T_{i j}=1 \mid Z_{i j}, m_{S_{i}}, a_{i}, m_{T_{i}}, b_{i}\right]\right)=\mu_{T}+m_{T_{i}}+\left(\beta+b_{i}\right) Z_{i j},
\end{array}\right.
$$

where $\Phi$ denotes the standard normal cumulative distribution function. Renard et al(34) used pseudolikelihood methods to estimate the model parameters. Similar ideas have been used in the case one of the endpoints is continuous, with the other one binary or categorical(4) (Ch. 6).

The case of two binary outcomes has recently received further attention, encompassing flexible software 
implementation, has been studied recently by Tilahun et al(35).

\subsection{Two Failure-time Endpoints}

Assume now that $S_{i j}$ and $T_{i j}$ are failure-time endpoints. Model (12)-(13) is replaced by a model for two correlated failure-time random variables. Burzykowski et al(40) used copulas to this end(36; 37). Precisely, one assumes the joint survivor function of $\left(S_{i j}, T_{i j}\right)$ is written as:

$$
F(s, t)=P\left(S_{i j} \geq s, T_{i j} \geq t\right)=C_{\delta}\left\{F_{S i j}(s), F_{T i j}(t)\right\}, \quad s, t \geq 0
$$

where $\left(F_{S i j}, F_{T i j}\right)$ denote marginal survivor functions and $C_{\delta}$ is a copula, i.e., a distribution function on $[0,1]^{2}$ with $\delta \in R^{1}$.

When the hazard functions are specified, estimates of the parameters for the joint model can be obtained using maximum likelihood. Shih and Louis(38) discuss alternative estimation methods. The association parameter is generally hard to interpret. However, it can be shown(39) that there is a link with Kendall's $\tau:$

$$
\tau=4 \int_{0}^{1} \int_{0}^{1} C_{\delta}(u, v) C_{\delta}(d u, d v)-1
$$

providing an easy measure of surrogacy at the individual level. At the second stage $R_{\text {trial }}^{2}$ can be computed based on the pairs of treatment effects estimated at the first stage.

\subsection{An Ordinal Surrogate and a Survival Endpoint}

Assume that $T$ is a failure-time random variable and $S$ is a categorical variable with $K$ ordered categories. To propose validation measures, similar to those introduced in the previous section, Burzykowski, Molenberghs, and Buyse(40) also used bivariate copulas, combining ideas of Molenberghs, Geys, and Buyse(41)and Burzykowski et al(40). One marginal distribution is a proportional odds logistic regression, while the other is a proportional hazards model. The Plackett copula(42) was chosen to capture the association between both endpoints. The ensuing global odds ratio is relatively easy to interpret.

\subsection{Methods for Combined Binary and Normally Distributed Endpoints}

Statistical problems where various outcomes of a combined nature are observed are common, especially with normally distributed outcomes on the one hand and binary or categorical outcomes on the other 
hand. Emphasis may be on the determination of the entire joint distribution of both outcomes or on specific aspects, such as the association in general or correlation in particular between both outcomes. Burzykowski, Molenberghs, and Buyse(4) review extensions of the meta-analytic approach, ranging over continuous, binary, ordinal, time-to-event, and longitudinally measured outcomes. Here, we focus on the combination of continuous and binary outcomes.

In this section, we start with a bivariate non-hierarchical setting, which can always be expressed as the product of a marginal distribution of one of the responses and the conditional distribution of the remaining response given the former one. The main problem with this approach is that no easy expressions for the association between both endpoints are available. Thus, we opt for a symmetric treatment of both endpoints. We focus on the case where the true endpoint is continuous and the surrogate is binary, the reverse case being entirely similar.

Generalized linear mixed models for endpoints of different data types are challenging(43). Hence, we concentrate on two-stage fixed-effects models. In the first stage, let $\widetilde{S}_{i j}$ be a latent variable of which $S_{i j}$ is the dichotomized version. A bivariate normal model for $\widetilde{S}_{i j}$ and $T_{i j}$ is given by(41):

$$
\begin{aligned}
& \widetilde{S}_{i j}=\mu_{S i}+\alpha_{i} Z_{i j}+\varepsilon_{S i j}, \\
& T_{i j}=\mu_{T i}+\beta_{i} Z_{i j}+\varepsilon_{T i j},
\end{aligned}
$$

where $\mu_{S i}$ and $\mu_{T i}$ are trial-specific intercepts, $\alpha_{i}$ and $\beta_{i}$ are trial-specific effects of treatment $Z_{i j}$ on the endpoints in trial $i$, and $\varepsilon_{S i}$ and $\varepsilon_{T i}$ are correlated error terms, assumed to be zero-mean normally distributed with covariance matrix

$$
\Sigma=\left(\begin{array}{cc}
\frac{1}{\left(1-\rho^{2}\right)} & \frac{\rho \sigma}{\sqrt{ }\left(1-\rho^{2}\right)} \\
& \sigma
\end{array}\right),
$$

where $\sigma$ is the variance of the continuous outcome and $\rho$ is the correlation between both outcomes. The variance of $\widetilde{S}_{i j}$ is chosen for computational reasons. Using a probit formulation like Molenberghs Geys, and Buyse(41) and owing to the replication at the trial level, we can impose a distribution on the trial-specific parameters. At the second stage, we assume

$$
\left(\begin{array}{c}
\mu_{S i} \\
\mu_{T i} \\
\alpha_{i} \\
\beta_{i}
\end{array}\right)=\left(\begin{array}{c}
\mu_{S} \\
\mu_{T} \\
\alpha \\
\beta
\end{array}\right)+\left(\begin{array}{c}
m_{S i} \\
m_{T i} \\
a_{i} \\
b_{i}
\end{array}\right),
$$


where the second term on the right hand of $(28)$ is assumed to follow a zero-mean normal distribution with dispersion matrix (16). Measures to assess the quality of the surrogate both at the trial and individual level are then obtained. This case has received full attention in Assam et al(44).

\subsection{Longitudinal Endpoints}

Most of the previous work focuses on univariate responses. Alonso et al(45) showed that going from a univariate setting to a multivariate framework represents new challenges. The $R^{2}$ measures proposed by Buyse et al(3), are no longer applicable. Alonso et al(45) based their calculations of surrogacy measures on a two-stage approach rather than a full random effects approach. They assume that information from $i=1, \ldots, N$ trials is available, in the $i$ th of which, $j=1, \ldots, n_{i}$ subjects are enrolled and they denoted the time at which subject $j$ in trial $i$ is measured as $t_{i j k}$. If $T_{i j k}$ and $S_{i j k}$ denote the associated true and surrogate endpoints, respectively, and $Z_{i j}$ is a binary indicator variable for treatment then along the ideas of Galecki(46), they proposed the following joint model, at the first stage, for both responses

$$
\left\{\begin{array}{l}
T_{i j k}=\mu_{T i}+\beta_{i} Z_{i j}+g_{T i j}\left(t_{i j k}\right)+\varepsilon_{T i j k}, \\
S_{i j k}=\mu_{S i}+\alpha_{i} Z_{i j}+g_{S i j}\left(t_{i j k}\right)+\varepsilon_{S i j k},
\end{array}\right.
$$

where $\mu_{T i}$ and $\mu_{S i}$ are trial-specific intercepts, $\beta_{i}$ and $\alpha_{i}$ are trial-specific effects of treatment $Z_{i j}$ on the two endpoints and $g_{T i j}$ and $g_{S i j}$ are trial-subject-specific time functions that can include treatment-bytime interactions. They also assume that the vectors, collecting all information over time for patient $j$ in trial $i, \widetilde{\varepsilon}_{T_{i j}}$ and $\widetilde{\varepsilon}_{S_{i j}}$ are correlated error terms, following a mean-zero multivariate normal distribution with covariance matrix

$$
\Sigma_{i}=\left(\begin{array}{cc}
\Sigma_{T T i} & \Sigma_{T S i} \\
\Sigma_{T S i}^{\prime} & \Sigma_{S S i}
\end{array}\right)=\left(\begin{array}{cc}
\sigma_{T T i} & \sigma_{T S i} \\
\sigma_{T S i} & \sigma_{S S i}
\end{array}\right) \otimes R_{i} .
$$

Here, $R_{i}$ is a correlation matrix for the repeated measurements.

If treatment effect can be assumed constant over time, then (19) can still be useful to evaluate surrogacy at the trial level. However, at the individual level the situation is totally different, the $R_{\text {ind }}^{2}$ no longer being applicable, and new concepts are needed.

Using multivariate ideas, Alonso et al(45) proposed the variance reduction factor $(V R F)$ to capture individual-level surrogacy in this more elaborate setting. They quantified the relative reduction in the 
true endpoint variance after adjustment by the surrogate as

$$
V R F_{\text {ind }}=\frac{\sum_{i}\left\{\operatorname{tr}\left(\Sigma_{T T i}\right)-\operatorname{tr}\left(\Sigma_{(T \mid S) i}\right)\right\}}{\sum_{i} \operatorname{tr}\left(\Sigma_{T T i}\right)},
$$

where $\Sigma_{(T \mid S)_{i}}$ denotes the conditional variance-covariance matrix of $\widetilde{\varepsilon}_{T_{i j}}$ given $\widetilde{\varepsilon}_{S_{i j}}: \Sigma_{(T \mid S) i}=\Sigma_{T T i}-$ $\Sigma_{T S i} \Sigma_{S S i}^{-1} \Sigma_{T S i}^{\prime}$. Here, $\Sigma_{T T i}$ and $\Sigma_{S S i}$ are the variance-covariance matrices associated with the true and surrogate endpoint respectively and $\Sigma_{T S i}$ contains the covariances between the surrogate and the true endpoint. Alonso et al(45) showed that the $V R F_{\text {ind }}$ ranges between zero and one, and that $V R F_{\text {ind }}=R_{\text {ind }}^{2}$ when the endpoints are measured only once.

An alternative proposal is

$$
\theta_{p}=\sum_{i} \frac{1}{N p_{i}} \operatorname{tr}\left\{\left(\Sigma_{T T i}-\Sigma_{(T \mid S) i}\right) \Sigma_{T T i}^{-1}\right\}
$$

Structurally, both $V R F$ and $\theta_{p}$ are similar, the difference being the reversal of summing the trace and calculating the ratio. In spite of this strong structural similarity the VRF is not symmetric in $S$ and $T$ and it is only invariant with respect to linear orthogonal transformations, whereas $\theta_{p}$ is both symmetric and invariant with respect to the broader class of linear bijective transformations.

A common problem of all previous proposals is that they are strongly based on the normality assumption and extensions to non-normal settings are difficult. To overcome this limitation, Alonso et al(47), introduced a new parameter, the so-called $R_{\Lambda}^{2}$, to evaluate surrogacy at the individual level when both responses are measured over time or in general when multivariate or repeated measures are available

$$
R_{\Lambda}^{2}=\frac{1}{N} \sum_{i}\left(1-\Lambda_{i}\right)
$$

where: $\Lambda_{i}=\frac{\left|\Sigma_{i}\right|}{\left|\Sigma_{T T i}\right|\left|\Sigma_{S S i}\right|}$. This parameter not only allows the detection of more general patterns of association but can also be extended to more general settings than those defined by the normal distribution. They proved that $R_{\Lambda}^{2}$ ranges between zero and one, and that in the cross-sectional case $R_{\Lambda}^{2}=R_{\text {ind }}^{2}$. These authors have shown that $R_{\Lambda}^{2}=1$ whenever there is a deterministic relationship between two linear combinations of both endpoints, allowing the detection of strong associations in cases where the VRF or $\theta_{p}$ would fail in doing so. 


\section{A Unified Approach}

The longitudinal method of the previous section, while elegant, hinges upon normality of the outcome. First using the likelihood reduction factor (Section 6.1) and then an information-theoretic approach (Section 6.2), extension, and therefore unification, will be achieved.

\subsection{The Likelihood Reduction Factor}

Estimating individual-level surrogacy, as the previous developments clearly show, has frequently been based on a variance-covariance matrix coming from the distribution of the residuals. However, if we move away from the normal distribution, it is not always clear how to quantify the association between both endpoints after adjusting for treatment and trial effect. To address this problem, Alonso et al(47) and Alonso and Molenberghs(48) considered the following generalized linear models

$$
\begin{aligned}
g_{T}\left\{E\left(T_{i j}\right)\right\} & =\mu_{T i}+\beta_{i} Z_{i j}, \\
g_{T}\left\{E\left(T_{i j} \mid S_{i j}\right)\right\} & =\theta_{0 i}+\theta_{1 i} Z_{i j}+\theta_{2 i} S_{i j},
\end{aligned}
$$

where $g_{T}$ is an appropriate link function, $\mu_{T i}$ are the trial-specific intercepts and $\beta_{i}$ are trial-specific effects of treatment $Z$ on the true endpoint in trial $i . \theta_{0 i}$ and $\theta_{1 i}$ are trial-specific intercepts and effects of treatment on the true endpoint when the surrogate endpoint is known. Note that (34) and (35) can be readily extended to incorporate more complex settings. Other extensions, such as non-linearity between $S_{i j}$ and $g_{T}\left\{E\left(T_{i j}\right)\right\}$ are possible. We assume a linear relationship between $S_{i j}$ and $g_{T}\left\{E\left(T_{i j}\right)\right\}$, but consider extensions of (34) and (35) in the light of simplified modeling strategy, as presented by Tibaldi et al(32). They suggested several simplifications for the case of continuous true and surrogate endpoints. They have introduced the concept of three possible dimensions along which simplifications can be made: the trial, endpoint, and measurement error dimensions. Their ideas can be applied outside the original mixed model based framework. We consider their trial and measurement error dimensions.

The trial dimension provides a choice between treating the trial-specific effects as fixed or random. The former is often chosen out of necessity, when the latter is too challenging. If the trial-specific effects are chosen fixed, then (34) and (35) are used to validate the surrogate endpoint. On the other hand, if the trial-specific effects are considered random, we extend (34) and (35) to appropriate generalized 
linear mixed-effects models

$$
\begin{aligned}
g_{T}\left\{E\left(T_{i j}\right)\right\} & =\mu_{T}+m_{T i}+\beta Z_{i j}+b_{i} Z_{i j}, \\
g_{T}\left\{E\left(T_{i j} \mid S_{i j}\right)\right\} & =\theta_{0}+c_{T i}+\theta_{1} Z_{i j}+a_{i} Z_{i j}+\theta_{2 i} S_{i j},
\end{aligned}
$$

where $\mu_{T}$ and $\beta$ are a fixed intercept and treatment effect on the true endpoint, while $m_{T i}$ and $b_{i}$ are a random intercept and treatment effects on the true endpoint. $\theta_{0}$ and $\theta_{1}$ are a fixed intercept and treatment effect on the true endpoint when the surrogate is known, and $c_{T i}$ and $a_{i}$ are a random intercept and treatment effects on the true endpoint when the surrogate is known.

It is often the case in practice that different trials in meta-analysis have different sizes. Since univariate models are used to evaluate surrogacy in the information-theoretic approach, there is a need to adjust for the heterogeneity in information content between trial-specific contributions. This is the target of the choices along the so-called measurement error dimension. One way to account for a variable amount of information per trial is by weighting the contributions according to trial size, thus giving rise to a weighted linear regression models, particularly when estimating measures for trial-level surrogacy.

Let us turn to the so-called likelihood reduction factor (LRF). Observe that, in the case where the true endpoint is continuous and normally distributed, (34) and (35) reduce to normal regression models and (36) and (37) reduce to linear mixed models. On the other hand, when the true endpoint is binary, (34) and (35) reduce to logistic regression models. Alonso and Molenberghs (2007) used the LRF to evaluate individual level surrogacy, which is obtained by

$$
L R F=1-\frac{1}{N} \sum_{i} \exp \left(-\frac{G_{i}^{2}}{n_{i}}\right)
$$

where $G_{i}^{2}$ denotes the log-likelihood ratio test statistic to compare (34) and (35) or (36) and (37) within trial $i$. Alonso et al (2005) established a number of properties for LRF, in particular its ranging in the unit interval and, importantly, its reduction to $R_{\text {ind }}^{2}$ in the cross-sectional case.

\subsection{An Information-theoretic Unification}

This proposal avoids the needs for a joint, hierarchical model, and allows for unification across different types of endpoints. The entropy of a random variable(49), a good measure of randomness or uncertainty, is defined in the following way for the case of a discrete random variable $Y$, taking values 
$\left\{k_{1}, k_{2}, \ldots, k_{m}\right\}$, and with probability function $P\left(Y=k_{i}\right)=p_{i}$ :

$$
H(Y)=\sum_{i} p_{i} \log \left(\frac{1}{p_{i}}\right)
$$

The differential entropy $h_{d}(X)$ of a continuous variable $X$ with density $f_{X}(x)$ and support $S_{f_{X}}$ equals

$$
h_{d}(X)=-E\left[\log f_{X}(X)\right]=-\int_{S_{f_{X}}} f_{X}(x) \log f_{X}(x) d x .
$$

The joint and conditional (differential) entropies are defined in an analogous fashion. Defining the information of a single event as $I(A)=\log p_{A}$, the entropy is $H(A)=-I(A)$. No information is gained from a totally certain event, $p_{A} \approx 1$, so $I(A) \approx 0$, while an improbable event is informative.

$H(Y)$ is the average uncertainty associated with $P$. Entropy is always non-negative, satisfies $H(Y \mid X) \leq$ $H(Y)$ for any pair of random variables, with equality holding under independence, and is invariant under a bijective transformation(50). Differential entropy enjoys some but not all properties of entropy: it can be infinitely large, negative, or positive, and is coordinate dependent. For a bijective transformation $Y=y(X)$, it follows $h_{d}(Y)=h_{d}(X)-\mathrm{E}_{Y}\left(\log \left|\frac{d x}{d y}(y)\right|\right)$.

We can now quantify the amount of uncertainty in $Y$, expected to be removed if the value of $X$ were known, by $I(X, Y)=h_{d}(Y)-h_{d}(Y \mid X)$, the so-called mutual information. It is always non-negative, zero if and only if $X$ and $Y$ are independent, symmetric, invariant under bijective transformations of $X$ and $Y$, and $I(X, X)=h_{d}(X)$. The mutual information measures the information of $X$, shared by $Y$.

We will now introduce the entropy-power(49) for comparison of continuous random variables. Let $X$ be a continuous $n$-dimensional random vector. The entropy-power of $X$ is

$$
\mathrm{EP}(X)=\frac{1}{(2 \pi e)^{n}} e^{2 h(X)}
$$

The differential entropy of a continuous normal random variable is $h(X)=\frac{1}{2} \log \left(2 \pi \sigma^{2}\right)$, a simple function of the variance and, on the natural logarithmic scale: $\operatorname{EP}(X)=\sigma^{2}$. In general, $\operatorname{EP}(X) \leq$ $\operatorname{Var}(X)$ with equality if and only if $X$ is normally distributed.

We can now define an information-theoretic measure of association(51):

$$
R_{h}^{2}=\frac{\operatorname{EP}(Y)-\operatorname{EP}(Y \mid X)}{\operatorname{EP}(Y)}
$$


which ranges in the unit interval, equals zero if and only if $(X, Y)$ are independent, is symmetric, is invariant under bijective transformation of $X$ and $Y$, and, when $R_{h}^{2} \rightarrow 1$ for continuous models, there is usually some degeneracy appearing in the distribution of $(\mathrm{X}, \mathrm{Y})$. There is a direct link between $R_{h}^{2}$ and the mutual information: $R_{h}^{2}=1-e^{-2 I(X, Y)}$. For $Y$ discrete: $R_{h}^{2} \leq 1-e^{-2 H(Y)}$, implying that $R_{h}^{2}$ then has an upper bound smaller than 1 ; we then redefine

$$
\widetilde{R}_{h}^{2}=\frac{R_{h}^{2}}{1-e^{-2 H(Y)}}
$$

reaching 1 when both endpoints are deterministically related.

We can now redefine surrogacy, while preserving previous proposals as special cases. While we will focus on individual-level surrogacy, all results apply to the trial level too. Let $Y=T$ and $X=S$ be the true and surrogate endpoints, respectively. We consider $S$ a good surrogate for $T$ at the individual (trial) level, if a "large" amount of uncertainty about $T$ (the treatment effect on $T$ ) is reduced when $S$ (the treatment effect on $S$ ) is known. Equivalently, we term $S$ a good surrogate for $T$ at the individual level, if our lack of knowledge about the true endpoint is substantially reduced when the surrogate endpoint is known.

A meta-analytic framework, with $N$ clinical trials, produces $N_{q}$ different $R_{h i}^{2}$, and hence we propose a meta-analytic $R_{h}^{2}$ :

$$
R_{h}^{2}=\sum_{i=1}^{N_{q}} \alpha_{i} R_{h i}^{2}=1-\sum_{i=1}^{N_{q}} \alpha_{i} e^{-2 I_{i}\left(S_{i}, T_{i}\right)},
$$

where $\alpha_{i}>0$ for all $i$ and $\sum_{i=1}^{N_{q}} \alpha_{i}=1$. Different choices for $\alpha_{i}$ lead to different proposals, producing an uncountable family of parameters. This opens the additional issue of finding an optimal choice. In particular, for the cross-sectional normal-normal case, Alonso and Molenberghs (2007) have shown that $R_{h}^{2}=R_{\text {ind }}^{2}$. The same holds for $R_{\Lambda}^{2}$ for the longitudinal case. Finally, when the true and surrogate endpoints have distributions in the exponential family, then LRF $\stackrel{P}{\rightarrow} R_{h}^{2}$ when the number of subjects per trial goes to infinity.

Alonso and Molenberghs(48) developed asymptotic confidence intervals for $R_{h}^{2}$, based on the idea of(52), to build confidence intervals for $2 I(T, S)$. Let $\hat{a}=2 n \widehat{I}(T, S)$, where $n$ is the number of patients. Define $\kappa_{1: \alpha}(\mathrm{a})$ and $\delta_{1: \alpha}(\mathrm{a})$ by $P\left(\chi_{1}^{2}\left(\kappa_{1: \alpha}(a)\right) \geq a\right)=\alpha$ and $P\left(\chi_{1}^{2}\left(\delta_{1: \delta}(a)\right) \leq a\right)=\alpha$. Here, $\chi_{1}^{2}$ is a chisquared random variable with 1 degree of freedom. If $P\left(\chi_{1}^{2}(0) \geq a\right)=\alpha$ then we set $\kappa_{1: \alpha}(a)=0$. A 
conservative two-sided $1-\alpha$ asymptotic confidence interval for $R_{h}^{2}$ is

$$
\sum_{i} \alpha_{i}\left[n_{i}^{-1} \kappa_{1: \alpha}^{i}(\hat{a}), n_{i}^{-1} \delta_{1: \alpha}^{i}(\hat{a})\right]
$$

where $1-\alpha_{i}$ is the Bonferroni confidence level for the trial intervals (Alonso and Molenberghs 2007). This asymptotic interval has considerable computational advantage with respect to the bootstrap approach used by Alonso et al (2005). Although ITA involves substantial mathematics, its implementation in practice is fairly straightforward and less computer-intensive than the meta-analytic approach. This is a direct consequence of the fact that the models used in the former are univariate models, which can be fitted using any standard regression software. However, the performance of this approach has not been studied in the mixed continuous and binary endpoint settings. In the next section, insight into the performance of this approach, together with that of the asymptotic interval, is offered through a simulation study.

\subsection{Fano's Inequality and the Theoretical Plausibility of Finding a Good Surrogate}

Fano's inequality shows the relationship between entropy and prediction:

$$
\mathrm{E}\left[(T-g(S))^{2}\right] \geq \operatorname{EP}(T)\left(1-R_{h}^{2}\right)
$$

where $\operatorname{EP}(T)=\frac{1}{2 \pi e} e^{2 h(T)}$. Note that nothing has been assumed about the distribution of our responses and no specific form has been considered for the prediction function $g$. Also, (44) shows that the predictive quality strongly depends on the characteristics of the endpoint, specifically on its powerentropy. Fano's inequality states that the prediction error increases with $\operatorname{EP}(T)$ and therefore, if our endpoint has a large power-entropy then a surrogate should produce a large $R_{h}^{2}$ to have some predictive value. This means that, for some endpoints, the search for a good surrogate can be a dead end street: the larger the entropy of $T$ the more difficult it is to predict. Studying the power-entropy before trying to find a surrogate is therefore advisable.

\subsection{Application to the Meta-analysis of Five Clinical Trials in Schizophrenia}

We will treat $\mathrm{CGI}$ as the true endpoint and PANSS as surrogate, although the reverse would be sensible, too. In practice, these endpoints are frequently dichotomized in a clinically meaningful way. Our binary true endpoint $T=$ CGId $=1$ for patients classified from "Very much improved" to "Improved", and 0 
otherwise. The binary surrogate $S=$ PANSSd $=1$ for patients with at least 20 points reduction versus baseline, and 0 otherwise. We will start from probit and Plackett-Dale models and compare results with the ones from the information-theoretic approach.

In line with Section 5.1, we formulate two continuous latent variables $\left(\widetilde{\mathrm{CGI}}_{i j}, \widehat{\mathrm{PANSS}}_{i j}\right)$ assumed to follow a bivariate normal distribution. The following probit model can be fitted

$$
\left(\begin{array}{c}
\widetilde{\mu}_{i j}^{T} \\
\widetilde{\mu}_{i j}^{S} \\
\ln \left(\sigma^{2}\right) \\
\ln \left(\frac{1+\widetilde{\rho}}{1+\widetilde{\rho}}\right)
\end{array}\right)=\left(\begin{array}{c}
\widetilde{\mu}_{T_{i}}+\widetilde{\beta}_{i} Z_{i j} \\
\widetilde{\mu}_{S_{i}}+\widetilde{\alpha}_{i} Z_{i j} \\
c_{\sigma^{2}} \\
c_{\widetilde{\rho}}
\end{array}\right)
$$

where $\widetilde{\mu}_{i j}^{T}=E\left(\widetilde{\mathrm{CG}}_{i j}\right), \quad \tilde{\mu}_{i j}^{S}=E\left(\widetilde{\operatorname{PANSS}}_{i j}\right), \quad \operatorname{Var}\left(\widetilde{\mathrm{CGI}}_{i j}\right)=1, \quad \sigma^{2}=\operatorname{Var}\left(\widetilde{\mathrm{PANSS}}_{i j}\right)$ and $\tilde{\rho}=$ $\operatorname{corr}\left(\widetilde{\mathrm{CG}}_{i j}, \widetilde{\mathrm{PANSS}}_{i j}\right)$ denotes the correlation between the true and surrogate endpoint latent variables. We can then use the estimated values of $\left(\widetilde{\mu}_{S_{i}}, \widetilde{\alpha}_{i}, \widetilde{\beta}_{i}\right)$ to evaluate trial level surrogacy through the $R_{\text {trial }}^{2}$. At the individual level, $\widetilde{\rho}^{2}$ is used to capture surrogacy.

Alternatively, the Dale(42) formulation can be used, based on

$$
\left(\begin{array}{c}
\operatorname{logit}\left(\pi_{i j}^{T}\right) \\
\operatorname{logit}\left(\pi_{i j}^{S}\right) \\
\ln (\psi)
\end{array}\right)=\left(\begin{array}{c}
\mu_{T_{i}}+\beta_{i} Z_{i j} \\
\mu_{S_{i}}+\alpha_{i} Z_{i j} \\
c_{\psi}
\end{array}\right)
$$

where $\pi_{i j}^{T}=\mathrm{E}\left(\operatorname{CGld}_{i j}\right), \pi_{i j}^{S}=\mathrm{E}\left(\operatorname{PANSSd}_{i j}\right)$ and $\psi$ is the global odds ratio associated to both endpoint. As before, the estimated values of $\left(\mu_{S_{i}}, \alpha_{i}, \beta_{i}\right)$ can be used to evaluate surrogacy at the trial level and the individual level surrogacy is quantified using the global odds ratio.

In the information-theoretic approach the following three models are fitted independently

$$
\begin{aligned}
\Phi\left(\pi_{i j}^{T}\right) & =\mu_{T_{i}}+\beta_{i} Z_{i j}, \\
\Phi\left(\pi_{i j}^{T \mid S}\right) & =\mu_{T_{i}}^{S}+\beta_{i}^{S} Z_{i j}+\gamma_{i j} S_{i j}, \\
\Phi\left(\pi_{i j}^{S}\right) & =\mu_{S_{i}}+\alpha_{i} Z_{i j},
\end{aligned}
$$

where $\pi_{i j}^{T}=\mathrm{E}\left(\operatorname{CGld}_{i j}\right), \pi_{i j}^{T \mid S}=\mathrm{E}\left(\operatorname{CGld}_{i j} \mid \operatorname{PANSSd}_{i j}\right), \pi_{i j}^{S}=\mathrm{E}\left(\operatorname{PANSSd}_{i j}\right)$ and $\Phi$ denotes the cumulative standard normal distribution. At the trial level, the estimated values of $\left(\mu_{S_{i}}, \alpha_{i}, \beta_{i}\right)$ obtained from (47) and (49) can be used to calculate the $R_{\text {trial' }}^{2}$, whereas at the individual level we can quantify surrogacy 
using $R_{h}^{2}$. As it was stated before, the LRF is a consistent estimator of $R_{h}^{2}$, however, in principle other estimators could be used as well. We will then quantify surrogacy at the individual level by $\hat{R}_{h}^{2}=1-\exp \left(-G^{2} / n\right)$, where $G^{2}$ is the loglikelihood ratio test to compare (47) with (48) and $n$ denotes total number of patients. Furthermore, when applied to the binary-binary setting, Fanos's inequality takes the form

$$
P(T \neq S) \geq \frac{1}{\log |\Psi|}\left[H(T)-1+\frac{1}{2} \ln \left(1-R_{h}^{2}\right)\right]
$$

where $\Psi=\{0,1\}$ and $|\Psi|$ denotes the cardinal of $\Psi$. Here, again, Fano's inequality gives a lower bound for the probability of incorrect prediction.

Table 3 shows the results at the trial and individual level obtained with the different approaches described above. At the trial level, all the methods produced very similar values for the validation measure. In all cases, $R_{\text {trial }}^{2} \simeq 0.50$. It is also remarkable that the probit approach, in spite of being based on treatment effects defined at a latent level, produced a $R_{\text {trial }}^{2}$ value similar to the ones obtained with the information-theoretic and Plackett-Dale approaches. However, as Alonso et al (2003) showed, there is a linear relationship between the mean parameters defined at the latent level and the mean parameters of the model based on the observable endpoints and that could explain the agreement between the probit and the other two procedures. Therefore, at the trial level, we could conclude that knowing the treatment effect on the surrogate will reduce our uncertainty about the treatment effect on the true endpoint by $50 \%$.

At the individual level, the probit approach gives the strongest association between the surrogate and the true endpoint. Nevertheless, this value describes the association at an unobservable latent level, rendering its interpretation more awkward than with information theory, since it is not clear how this latent association could be relevant from a clinical point of view or how it could be translated into an association for the observable endpoints. The Plackett-Dale procedure quantifies surrogacy using a global odds ratio, making the comparison between this method and the others more difficult. Note that even though odds ratios are widely used in biomedical fields the lack of an upper bound makes difficult their interpretation in this setting.

On the other hand, the value of the $R_{h \max }^{2}$ illustrates that the surrogate can merely explain $39 \%$ of our uncertainty about the true endpoint, a relatively low value. Additionally, the lower bound for 
Fano's inequality clearly shows that using the value of PANSS to predict the outcome on CGI would be misleading in at least $8 \%$ of the cases. Even though this value is relatively low, it is only a lower bound and the real probability of mistake could be much larger.

At the trial level, the information-theoretic approach produces results similar to the ones from the conventional methods, but does so by means of models that are generally much easier to fit. At the individual level, the information-theoretic approach avoids the problem common with the probit model in that the correlation of the latter is formulated at the latent scale and therefore less relevant for practice. In addition, the information-theoretic measure ranges between 0 and 1 , circumventing interpretational problems arising from using the unbounded Plackett-Dale based odds ratio.

\section{$7 \quad$ Alternatives and Extensions}

Even for continuous outcomes, the conventional meta-analytic framework and its associated estimation methodology of a likelihood-based mixed-model nature, can pose computational challenges, while also the issues outlined in Section 4.3 need to be given proper consideration. As a result, several alternative strategies have been considered. For example, Shkedy and Torres Barbosa (2005) study in detail the use of Bayesian methodology and conclude that even relatively non-informative prior have a strongly beneficial impact on the algorithms' performance.

Cortiñas, Shkedy, and Molenberghs (2008) start from the information-theoretic approach, in the contexts of: (1) normally distributed endpoints; (2) a copula model for a categorical surrogate and a survival true endpoint; and (3) a joint modeling approach for longitudinal surrogate and true endpoints. Rather than fully relying on the methods described in Section 5, they use cross-validation to obtain adequate estimates of the trial-level surrogacy measure. Also, they explore the use of regression tree analysis, bagging regression analysis, random forests, and support vector machine methodology. They concluded that performance of such methods, in simulations and case studies, in terms of point and interval estimation, ranges from very good to excellent.

The above are variations to the meta-analytic theme, as described here, in Burzykowski, Molenberghs, and Buyse(4), and of which Daniels and Hughes (25) is an early instance. There are a number of alternative paradigms. Frangakis and Rubin(53) employ so-called principal stratification, still using the 
data from a single trial only. Drawing from the causality literature, Robins and Greenland(54), Pearl(55), and Taylor, Wang, and Thiébaut(56) use the direct/indirect-effect machinery.

\section{Prediction and Design Aspects}

Thus far, we have focused on quantifying surrogacy through a slate of measures, culminating in the information-theoretic ones. In practice, one may want to go beyond merely quantifying the strength of surrogacy, and further use a surrogate endpoint to predict the treatment effect on the true endpoint without measuring the latter. Put simply, the issue then is to obtain point and interval predictions for the treatment effect on the true endpoint based on the surrogate. This issue has been studied by Burzykowski and Buyse (2006) for the original meta-analytic approach for continuous endpoints and will be reviewed here.

The key motivation for validating a surrogate endpoint is the ability to predict the effect of treatment on the true endpoint based on the observed effect of treatment on the surrogate endpoint. It is essential, therefore, to explore the quality of prediction by (a) information obtained in the validation process based on trials $i=1, \ldots, N$ and (b) the estimate of the effect of $Z$ on $S$ in a new trial $i=0$. Fitting the mixed-effects model (12)-(13) to data from a meta-analysis provides estimates for the parameters and the variance components. Suppose then that a new trial $i=0$ is considered for which data are available on the surrogate endpoint but not on the true endpoint. We can then fit the following linear model to the surrogate outcomes $S_{0 j}$ :

$$
S_{0 j}=\mu_{S 0}+\alpha_{0} Z_{0 j}+\varepsilon_{S 0 j}
$$

We are interested in an estimate of the effect $\beta+b_{0}$ of $Z$ on $T$, given the effect of $Z$ on $S$. To this end, one can observe that $\left(\beta+b_{0} \mid m_{S 0}, a_{0}\right)$, where $m_{S 0}$ and $a_{0}$ are, respectively, the surrogate-specific random intercept and treatment effect in the new trial follows a normal distribution with mean linear in $\mu_{S 0}, \mu_{S}, \alpha_{0}$, and $\alpha$, and variance

$$
\operatorname{Var}\left(\beta+b_{0} \mid m_{S 0}, a_{0}\right)=\left(1-R_{\text {trial }}^{2}\right) \operatorname{Var}\left(b_{0}\right)
$$

Here, $\operatorname{Var}\left(b_{0}\right)$ denotes the unconditional variance of the trial-specific random effect, related to the effect of $Z$ on $T$ (in the past or the new trials). The smaller the conditional variance (51), the higher the precision of the prediction, as captured by $R_{\text {trial }}^{2}$. Let us use $\vartheta$ to group the fixed-effects parameters and 
variance components related to the mixed-effects model (12)-(13), with $\widehat{\vartheta}$ denoting the corresponding estimates. Fitting the linear model (50) to data on the surrogate endpoint from the new trial provides estimates for $m_{S 0}$ and $a_{0}$. The prediction variance can be written as:

$$
\operatorname{Var}\left(\beta+b_{0} \mid \mu_{S 0}, \alpha_{0}, \vartheta\right) \approx f\left\{\operatorname{Var}\left(\widehat{\mu}_{S 0}, \widehat{\alpha}_{0}\right)\right\}+f\{\operatorname{Var}(\widehat{\vartheta})\}+\left(1-R_{\text {trial }}^{2}\right) \operatorname{Var}\left(b_{0}\right)
$$

where $f\left\{\operatorname{Var}\left(\widehat{\mu}_{S 0}, \widehat{\alpha}_{0}\right)\right\}$ and $f\{\operatorname{Var}(\widehat{\vartheta})\}$ are functions of the asymptotic variance-covariance matrices of

$\left(\widehat{\mu}_{S 0}, \widehat{\alpha}_{0}\right)^{T}$ and $\widehat{\vartheta}$, respectively. The third term on the right hand side of (52), which is equivalent to (51), describes the prediction's variability if $\mu_{S 0}, \alpha_{0}$, and $\vartheta$ were known. The first two terms describe the contribution to the variability due to the use of the estimates of these parameters. It is useful to consider three scenarios.

Scenario 1. Estimation error in both the meta-analysis and the new trial. If the parameters of models (12)-(13) and (50) have to be estimated, as is the case in reality, the prediction variance is given by (52). From the equation it is clear that in practice, the reduction of the variability of the estimation of $\beta+b_{0}$, related to the use of the information on $m_{S 0}$ and $a_{0}$, will always be smaller than that indicated by $R_{\text {trial }}^{2}$. The latter coefficient can thus be thought of as measuring the "potential" validity of a surrogate endpoint at the trial-level, assuming precise knowledge (or infinite numbers of trials and sample sizes per trial available for the estimation) of the parameters of models (12)-(13) and (50). See also Scenario 3 below.

Scenario 2. Estimation error only in the meta-analysis. This scenario is possible only in theory, as it would require an infinite sample size in the new trial. But it can provide information of practical interest since, with an infinite sample size, the parameters of the single-trial regression model (50) would be known. Consequently, the first term on the right hand side of (52), $f\left\{\operatorname{Var}\left(\widehat{\mu}_{S 0}, \widehat{\alpha}_{0}\right)\right\}$, would vanish and (52) would reduce to

$$
\operatorname{Var}\left(\beta+b_{0} \mid \mu_{S 0}, \alpha_{0}, \vartheta\right) \approx f\{\operatorname{Var}(\widehat{\vartheta})\}+\left(1-R_{\text {trial }}^{2}\right) \operatorname{Var}\left(b_{0}\right)
$$

Expression (53) can thus be interpreted as indicating the minimum variance of the prediction of $\beta+b_{0}$, achievable in the actual application of the surrogate endpoint. In practice, the size of the meta-analytic data providing an estimate of $\vartheta$ will necessarily be finite and fixed. Consequently, the first term on the right hand side of (53) will always be present. Based on this observation, Gail et al(31) conclude that 
the use of surrogates validated through the meta-analytic approach will always be less efficient than the direct use of the true endpoint. Of course, even so, a surrogate can be of great use in terms of reduced sample size, reduce trial length, gain in number of life years, etc.

Scenario 3. No estimation error. If the parameters of the mixed-effects model (12)-(13) and the single-trial regression model (50) were known, the prediction variance for $\beta+b_{0}$ would only contain the last term on the right hand side of (52). Thus, the variance would be reduced to (51), which is clearly linked with (44). While this situation is, strictly speaking, of theoretical relevance only, as it would require infinite numbers of trials and sample sizes per trial available for the estimation in the meta-analysis and in the new trial, it provides important insight.

Based on the above scenarios one can argue that in a particular application the size of the minimum variance (53) is of importance. The reason is that (53) is associated with the minimum width of the prediction interval for $\beta+b_{0}$ that might be approached in a particular application by letting the sample size for the new trial increase towards infinity. This minimum width will be responsible for the loss of efficiency related to the use of the surrogate, pointed out in Gail et al(31). It would thus be important to quantify the loss of efficiency, since it may be counter-balanced by a shortening of trial duration. One might consider using the ratio of (53) to $\operatorname{Var}\left(b_{0}\right)$, the unconditional variance of $\beta+b_{0}$. However, Burzykowski and Buyse (2006) considered another way of expressing this information, which should be more meaningful clinically.

\subsection{Surrogate Threshold Effect}

We will outline the proposal made by Burzykowski and Buyse(57) and first focus on the case where the surrogate and true endpoints are jointly normally distributed. Assume that the prediction of $\beta+b_{0}$ can be made independently of $\mu_{S 0}$. Under this assumption the conditional mean of $\beta+b_{0}$ is a simple linear function of $\alpha_{0}$, the treatment effect on the surrogate, while the conditional variance can be written as

$$
\operatorname{Var}\left(\beta+b_{0} \mid \alpha_{0}, \vartheta\right)=\operatorname{Var}\left(b_{0}\right)\left(1-R_{\text {trial(r) }}^{2}\right)
$$

The coefficient of determination $R_{\text {trial(r) }}^{2}$ in (54) is simply the square of the correlation coefficient of trial-specific random effects $b_{i}$ and $a_{i}$. If $\vartheta$ were known and $\alpha_{0}$ could be observed without measurement error (i.e., assuming an infinite sample size for the new trial), the prediction variance would equal (54). 
In practice, an estimate $\widehat{\vartheta}$ is used and then prediction variance (53) ought to be applied:

$$
\operatorname{Var}\left(\beta+b_{0} \mid \alpha_{0}, \vartheta\right) \approx f\{\operatorname{Var}(\widehat{\vartheta})\}+\left(1-R_{\text {trial(r) }}^{2}\right) \operatorname{Var}\left(b_{0}\right)
$$

Since in linear mixed models the maximum likelihood estimates of the covariance parameters are asymptotically independent of the fixed effects parameters(16), one can show that the prediction variance (55) can be expressed approximately as a quadratic function of $\alpha_{0}$.

Let us consider a $(1-\gamma) 100 \%$ prediction interval for $\beta+b_{0}$ :

$$
E\left(\beta+b_{0} \mid \alpha_{0}, \vartheta\right) \pm z_{1-\frac{\gamma}{2}} \sqrt{\operatorname{Var}\left(\beta+b_{0} \mid \alpha_{0}, \vartheta\right)}
$$

where $z_{1-\gamma / 2}$ is the $(1-\gamma / 2)$ quantile of the standard normal distribution. The limits of the interval (56) are functions of $\alpha_{0}$. Define the lower and upper prediction limit functions of $\alpha_{0}$ as

$$
l\left(\alpha_{0}\right), u\left(\alpha_{0}\right) \equiv E\left(\beta+b_{0} \mid \alpha_{0}, \vartheta\right) \pm z_{1-\frac{\gamma}{2}} \sqrt{\operatorname{Var}\left(\beta+b_{0} \mid \alpha_{0}, \vartheta\right)} .
$$

One might then compute a value of $\alpha_{0}$ such that

$$
l\left(\alpha_{0}\right)=0 .
$$

Depending on the setting, one could also consider the upper limit $u\left(\alpha_{0}\right)$. We will call this value the surrogate threshold effect (STE). Its magnitude depends on the variance of the prediction. The larger the variance, the larger the absolute value of STE. From a clinical point of view, a large value of STE points to the need of observing a large treatment effect on the surrogate endpoint in order to conclude a non-zero effect on the true endpoint. In such a case, the use of the surrogate would not be reasonable, even if the surrogate were "potentially" valid, i.e., with $R_{\text {trial(r) }}^{2} \simeq 1$. The STE can thus provide additional important information about the usefulness of the surrogate in a particular application.

Note that the interval (56) and the prediction limit function $l\left(\alpha_{0}\right)$ can be constructed using the variances given by (54) or (55). Consequently, one might get two versions of STE. The version obtained from using (54) will be denoted by $\mathrm{STE}_{\infty, \infty}$. The infinity signs indicate that the measure assumes the knowledge of both of $\vartheta$ as well as of $\alpha_{0}$, achievable only with an infinite number of infinite-sample-size trials in the meta-analytic data and an infinite sample size for the new trial. In practice, $\mathrm{STE}_{\infty, \infty}$ will be computed using estimates. A large value of $\mathrm{STE}_{\infty, \infty}$ would point to the need of observing a large 
treatment effect on the surrogate endpoint even if there were no estimation error present. In this case, one would question even the "potential" validity of the surrogate.

If the variance (55) is used to define $l\left(\alpha_{0}\right)$, we will denote the STE by $\mathrm{STE}_{N, \infty}$, with $N$ indicating the need for the estimation of $\vartheta$. $\mathrm{STE}_{N, \infty}$ captures the "practical" validity of the surrogate, which accounts for the need of estimating parameters of model (12)-(13). It is possible that a surrogate might seem to be "potentially valid" (low $\mathrm{STE}_{\infty, \infty}$ value), but might not be valid "practically" (large $\mathrm{STE}_{N, \infty}$ value), owing to the loss of precision resulting from estimation of the mixed-effects model parameters. The roots of (58) can be obtained by solving a quadratic equation. The number of solutions of the equation depends on the parameter configuration in $l\left(\alpha_{0}\right)(57)$.

$\mathrm{STE}_{\infty, \infty}$ and $\mathrm{STE}_{N, \infty}$ can address concerns about the usefulness of the meta-analytic approach, expressed by Gail et al(31). They noted that, even for a valid surrogate, the variance of the prediction of treatment effect on the true endpoint cannot be reduced to 0 , even in the absence of any estimation error. $\mathrm{STE}_{N, \infty}$ can be used to quantify this loss of efficiency.

Interestingly, the STE can be expressed in terms of treatment effect on the surrogate necessary to be observed to predict a significant treatment effect on the true endpoint. In a practical application, one would seek a value of STE (preferably, $\mathrm{STE}_{N, \infty}$ ) well within the range of treatment effects on surrogates observed in previous clinical trials, as close as possible to the (weighted) mean effect.

STE and its estimation have been developed under the mixed-effects model (12)-(13), but Burzykowski and Buyse(57) also derived the STE when, perhaps for numerical convenience, the two-stage approach of Section 4.2 is used. Furthermore, STE can be computed for any type of surrogate. To this aim, one merely needs to use an appropriate joint model for surrogate and true endpoints, capable of providing the required treatment effect. Burzykowski and Buyse(57) presented time-to-event applications.

\section{Concluding Remarks}

Over the years, a variety of surrogate marker evaluation strategies have been proposed, cast within a meta-analytic framework. With an increasing range of endpoint types considered, such as continuous, binary, time-to-event, and longitudinal endpoints, also the scatter of types of measures proposed has increased. Some of these measures are difficult to calculate from fully specified hierarchical models, 
which has sparked off the formulation of simplified strategies. We reviewed the ensuing divergence of proposals, which then has triggered efforts of convergence, eventually leading to the informationtheoretic approach, which is both general and simple to implement. These developments have been illustrated using data from clinical trials in schizophrenia.

While quantifying surrogacy is important, so is prediction of the treatment effect in a new trial based on the surrogate. Work done in this area has been reviewed, with emphasis on the so-called surrogate threshold effect and the sources of variability involved in the prediction process. A connection with the information-theoretic approach is pointed out.

Even though more work is called for, we believe the information-theoretic approach and the surrogate threshold effect are promising paths towards effective assessment and use of surrogate endpoints in practice. Software implementations for methodology described here and beyond are available from www.uhasselt.be/censtat.

A key issue is whether a surrogate is still valid if, in a new trial, the same surrogate and true endpoints, but a different drug is envisaged. This is the so-called "class" question. It is usually argued that a surrogate could still be used if the new drug belongs to the same class of drugs as the ones in the evaluation exercise. Of course, this in itself is rather subjective and clinical expertise is necessary to meaningfully delineate a drug class.

\section{Acknowledgment}

The authors gratefully acknowledge support from Belgian IUAP/PAI network "Statistical Techniques and Modeling for Complex Substantive Questions with Complex Data".

\section{References}

[1] Prentice RL. Surrogate endpoints in clinical trials: definitions and operational criteria. Statistics in Medicine 1989; 8: 431-440.

[2] Freedman LS, Graubard BI, Schatzkin A. Statistical validation of intermediate endpoints for chronic diseases. Statistics in Medicine 1992; 11: 167-178. 
[3] Buyse M, Molenberghs G, Burzykowski T, Renard D, Geys H. The validation of surrogate endpoints in meta-analyses of randomized experiments. Biostatistics 2000; 1: 49-68.

[4] Burzykowski T, Molenberghs G, and Buyse M (2005). The Evaluation of Surrogate Endpoints. New York: Springer.

[5] Ellenberg SS, Hamilton JM. Surrogate endpoints in clinical trials: cancer. Statistics in Medicine 1989; 8: 405-413.

[6] Biomarkers Definition Working Group. Biomarkers and surrogate endpoints: preferred definitions and conceptual framework. Clinical Pharmacological Therapy 2001; 69: 89-95.

[7] Fleming TR, DeMets DL. Surrogate end points in clinical trials: are we being misled? Annals of Internal Medicine 1996; 125: 605-613.

[8] The Cardiac Arrhythmia Suppression Trial (CAST) Investigators. Preliminary Report: effect of encainide and flecainide on mortality in a randomized trial of arrhythmia suppression after myocardial infraction. New England Journal of Medicine 1989; 321: 406-412.

[9] DeGruttola V, Tu XM Modelling progression of CD-4 lymphocyte count and its relationship to survival time. Biometrics 1994; 50, 1003-1014.

[10] Lagakos SW, Hoth DF. Surrogate markers in AIDS: Where are we? Where are we going? Annals of Internal Medicine 1992; 116: 599-601.

[11] DeGruttola V, Fleming TR, Lin DY, Coombs R. Validating surrogate markers - are we being naive? Journal of Infectious Diseases 1997; 175: 237-246.

[12] Fleming TR. Surrogate markers in AIDS and cancer trials. Statistics in Medicine 1994; 13: 14231435.

[13] Ferentz AE. Integrating pharmacogenomics into drug development. Pharmacogenomics 2002; 3: 453-467.

[14] Lesko LJ, Atkinson AJ. Use of biomarkers and surrogate endpoints in drug development and regulatory decision making: criteria, validation, strategies. Annu Rev Pharmacological Toxicology 2001; 41: $347-66$. 
[15] Dunn N, Mann RD Prescription-event and other forms of epidemiological monitoring of side-effects in the UK. Clinical and Experimental Allergy 1999; 29: 217-239.

[16] Verbeke, G, Molenberghs G (2000). Linear Mixed Models for Longitudinal Data. New York: Springer.

[17] Jones TC. Call for a new approach to the process of clinical trials and drug registration. British Medical Journal 2001; 322: 920-923.

[18] Heise C, Sampson-Johannes A, Williams A, McCormick F, Von Hoff DD, Kirn DH. ONYX-015, an E1B gene-attenuated adenovirus, causes tumor-specific cytolysis and antitumoral efficacy that can be augmented by standard chemo-therapeutic agents. Nature Medicine 1997, 3: 639-645.

[19] Schatzkin A, Gail M. The promise and peril of surrogate end points in cancer research. Nature Reviews Cancer 2002; 2: 19-27.

[20] International Conference on harmonisation of technical requirements for registration of pharmaceuticals for human use. ICH Harmonised Tripartite Guideline. Statistical principles for clinical trials. (http://www.ich.org/pdflCH/e9.pdf), Federal Register 1998, 63, No. 179, 49583.

[21] Kay SR, Fiszbein A, Opler LA The positive and negative syndrome scale (PANSS) for schizophrenia. Schizophrenia Bulletin 1987; 13: 261-276.

[22] Kay SR, Opler LA, Lindenmayer JP. Reliability and validity of the Positive and Negative Syndrome Scale for schizophrenics. Psychiatric Research 1988; 23: 99-110.

[23] Joffe MM, Greene T. Related causal frameworks for surrogate outcomes. Biometrics 2008; 64; $1-10$.

[24] Buyse M, Molenberghs G. Criteria for the validation of surrogate end-points in randomized experiments. Biometrics 1998; 54: 1014-1029.

[25] Daniels MJ, Hughes MD. Meta-analysis for the evaluation of potential surrogate markers. Statistics in Medicine 1997; 16: 1515-1527. 
[26] Volberding PA, Lagakos SW, Koch, MA, et al. Zidovudine in asymptomtic human immunodeficiency virus infection: a controlled trial in persons with fewer than 500 CD4-positive cells per cubic millimeter. New England Journal of Medicine 1990; 322: 941-949.

[27] Choi S, Lagakos S, Schooley RT, Volberding PA. CD4+ lymphocytes are an incomplete surrogate marker for clinical progression in persons with asymptomatic HIV infection taking zidovudine. Annals of Internal Med 1993; 118: 674-680.

[28] Lin DY, Fleming TR, DeGruttola V. Estimating the proportion of treatment effect explained by a surrogate marker. Statistics in Medicine 1997; 16: 1515-1527.

[29] Flandre P, Saidi Y. Letter to the editor: estimating the proportion of treatment effect explained by a surrogate marker. Statistics in Medicine 1999; 18: 107-115.

[30] Begg C, Leung D. On the use of surrogate endpoints in randomized trials. Journal of the Royal Statistical Society, Series A 2000; 163: 26-27.

[31] Gail MH, Pfeiffer R, van Houwelingen HC, Carroll RJ. On meta-analytic assessment of surrogate outcomes. Biostatistics 2000; 1: 231-246.

[32] Tibaldi FS, Cortiñas Abrahantes J, Molenberghs G, Renard D, Burzykowski T, Buyse M, Parmar M, Stijnen T, Wolfinger R. Simplified hierarchical linear models for the evaluation of surrogate endpoints. Journal of Statistical Computation and Simulation 2003; 73: 643-658.

[33] Cortiñas Abrahantes J, Molenberghs G, Burzykowski T, Shkedy Z, Renard D. Choice of units of analysis and modeling strategies in multilevel hierarchical models. Computational Statistics and Data Analysis 2004; 47: 537-563.

[34] Renard D, Geys H, Molenberghs G, Burzykowski T, and Buyse M. Validation of surrogate endpoints in multiple randomized clinical trials with discrete outcomes. Biometrical Journal 2002; 44: 1-15.

[35] Tilahun A, Assam P, Alonso A, Molenberghs G. Information theory-based surrogate marker evaluation from several randomized clinical trials with binary endpoints, using SAS. Journal of Biopharmaceutical Statistics 2008; 18: 326-341. 
[36] Clayton DG. A model for association in bivariate life tables and its application in epidemiological studies of familial tendency in chronic disease incidence. Biometrika 1978; 65: 141-151.

[37] Hougaard P. Survival models for heterogeneous populations derived from stable distributions. Biometrika 1986; 73, 387-396.

[38] Shih JH, Louis TA. Inferences on association parameter in copula models for bivariate survival data. Biometrics 1995; 51: 1384-1399.

[39] Genest C, McKay J. The joy of copulas: bivariate distributions with uniform marginals. American Statistician 1986; 40, 280-283.

[40] Burzykowski T, Molenberghs G, and Buyse M. The validation of surrogate endpoints using data from randomized clinical trials: a case-study in advanced colorectal cancer. Journal of the Royal Statistical Society, Series A 2004; 167: 103-124.

[41] Molenberghs G, Geys H, Buyse M. Evaluation of surrogate end-points in randomized experiments with mixed discrete and continuous outcomes. Statist in Med 2001; 20: 3023-3038.

[42] Dale JR. Global cross ratio models for bivariate, discrete, ordered responses. Biometrics 1986; 42 : 909-917.

[43] Molenberghs G, and Verbeke, G. (2005). Models for Discrete Longitudinal Data. New York: Springer.

[44] Assam P, Tilahun A, Alonso A, Molenberghs G. Information-theory based surrogate marker evaluation from several randomized clinical trials with continuous true and binary surrogate endpoints. Clinical Trials 2007; 00: 000-000.

[45] Alonso A, Geys H, Molenberghs G, Vangeneugden T. Validation of surrogate markers in multiple randomized clinical trials with repeated measurements. Biometrical Journal 2003; 45: 931-945.

[46] Galecki A. General class of covariance structures for two or more repeated factors in longitudinal data analysis. Communications in Statistics: theory and methods 1994; 23: 3105-3119.

[47] Alonso A, Molenberghs G, Geys H, Buyse M. A unifying approach for surrogate marker validation based on Prentice's criteria. Statistics in Medicine 2005; 25: 205-211. 
[48] Alonso A, Molenberghs G. Surrogate marker evaluation from an information theoretic perspective. Biometrics 2007; 63: 180-186.

[49] Shannon C. A mathematical theory of communication, Bell System Technical Journal 1948; 27: 379-423 and 623-656.

[50] Cover, T. and Tomas, J. (1991). Elements of Information Theory. New York: Wiley.

[51] Schemper M, Stare J. Explained variation in survival analysis. Statistics in Medicine 1996; 15: 1999-2012.

[52] Kent, J. Information gain and a general measure of correlation. Biometrika 1983, 70, 163-173.

[53] Frangakis CE, Rubin DB. Principal stratification in causal inference. Biometrics 2004; 58: 21-29.

[54] Robins JM, Greenland S. Identifiability and exchangeability for direct and indirect effects. Epidemiology 1992; 3,: 143-155.

[55] Pearl, J. (2001). Causality: Models, Reasoning, and Inference. Cambridge: Cambridge University Press.

[56] Taylor JMG, Wang Y, Thiébaut R. Counterfactual links to the proportion of treatment effect explained by a surrogate marker. Biometrics 2005; 61: 1102-1111.

[57] Burzykowski T, Buyse M. Surrogate threshold effect: an alternative measure for meta-analytic surrogate endpoint validation. Pharmaceutical Statistics 2006; 5: 173-186. 
Table 1: Schizophrenia study. Results of the trial-level $\left(R_{\text {trial }}^{2}\right)$ surrogacy analysis.

\begin{tabular}{|c|c|c|c|c|}
\hline \multirow[b]{2}{*}{ Unit of analysis } & \multicolumn{2}{|c|}{ Fixed effects } & \multicolumn{2}{|c|}{ Random effects } \\
\hline & Unweighted & Weighted & Unweighted & Weighted \\
\hline \multicolumn{5}{|c|}{ Full Model } \\
\hline \multicolumn{5}{|c|}{ Univariate approach } \\
\hline Investigator & 0.5887 & 0.5608 & 0.5488 & 0.5447 \\
\hline Trial & 0.9641 & 0.9636 & 0.9849 & 0.9909 \\
\hline \multicolumn{5}{|c|}{ Bivariate approach } \\
\hline Investigator & 0.5887 & 0.5608 & \multicolumn{2}{|c|}{ 0.9898* } \\
\hline Trial & 0.9641 & 0.9636 & \multicolumn{2}{|c|}{ - } \\
\hline \multicolumn{5}{|c|}{ Reduced Model } \\
\hline \multicolumn{5}{|c|}{ Univariate approach } \\
\hline Investigator & 0.6707 & 0.5927 & 0.5392 & 0.5354 \\
\hline Trial & 0.8910 & 0.8519 & 0.7778 & 0.8487 \\
\hline \multicolumn{5}{|c|}{ Bivariate approach } \\
\hline Investigator & 0.6707 & 0.5927 & \multicolumn{2}{|c|}{$0.9999 *$} \\
\hline Trial & 0.7418 & 0.8367 & \multicolumn{2}{|c|}{$0.9999 *$} \\
\hline
\end{tabular}

*: The variance-covariance matrix is ill-conditioned; in particular, at least one eigenvalue is very close to zero. The condition numbers for the three models with ill-condition matrices, from top to bottom are $3.415 E+18,2.384 E+18$ and $1.563 E+18$ respectively.

Table 2: Examples of possible surrogate endpoints in various diseases (Abbreviations: AIDS = acquired immune deficiency syndrome; $A R M D=$ age-related macular degeneration; HIV = human immunodeficiency virus).

\begin{tabular}{lllll}
\hline \hline Disease & Surrogate Endpoint & Type & Final Endpoint & Type \\
\hline Resectable solid tumor & Time to recurrence & Censored & Survival & Censored \\
Advanced cancer & Tumor response & Binary & Time to progression & Censored \\
Osteoporosis & Bone mineral density & Longitudinal & Fracture & Binary \\
Cardiovascular disease & Ejection fraction & Continuous & Myocardial infraction & Binary \\
Hypertension & Blood pressure & Longitudinal & Coronary heart disease & Binary \\
Arrhythmia & Arrhythmic episodes & Longitudinal & Survival & Censored \\
ARMD & 6-month visual acuity & Continuous & 24-month visual acuity & Continuous \\
Glaucoma & Intraoccular pressure & Continuous & Vision loss & Censored \\
Depression & Biomarkers & Multivariate & Depression scale & Continuous \\
HIV infection & CD4 counts + viral load & Multivariate & Progression to AIDS & Censored \\
\hline \hline
\end{tabular}


Table 3: Schizophrenia study. Trial-level and individual-level validation measures (95\% confidence intervals). Binary-binary case.

\begin{tabular}{lcc}
\hline \hline Parameter & Estimate & $95 \%$ C.I. \\
\hline \multicolumn{3}{c}{ Trial-level $R_{\text {trial }}^{2}$ measures } \\
\hline 1.1 Information-theoretic & 0.49 & $(0.21,0.81)$ \\
1.2 Probit & 0.51 & $(0.18,0.78)$ \\
1.3 Plackett-Dale & 0.51 & $(0.21,0.81)$ \\
\hline \multicolumn{4}{c}{ Individual-level measures } \\
\hline$R_{h}^{2}$ & 0.27 & $(0.24,0.33)$ \\
$R_{h \text { max }}^{2}$ & 0.39 & $(0.35,0.48)$ \\
Probit & 0.67 & $(0.55,0.76)$ \\
Plackett-Dale $\psi$ & 25.12 & $(14.66 ; 43.02)$ \\
\hline Fano's lower-bound & 0.08 \\
\hline \hline
\end{tabular}



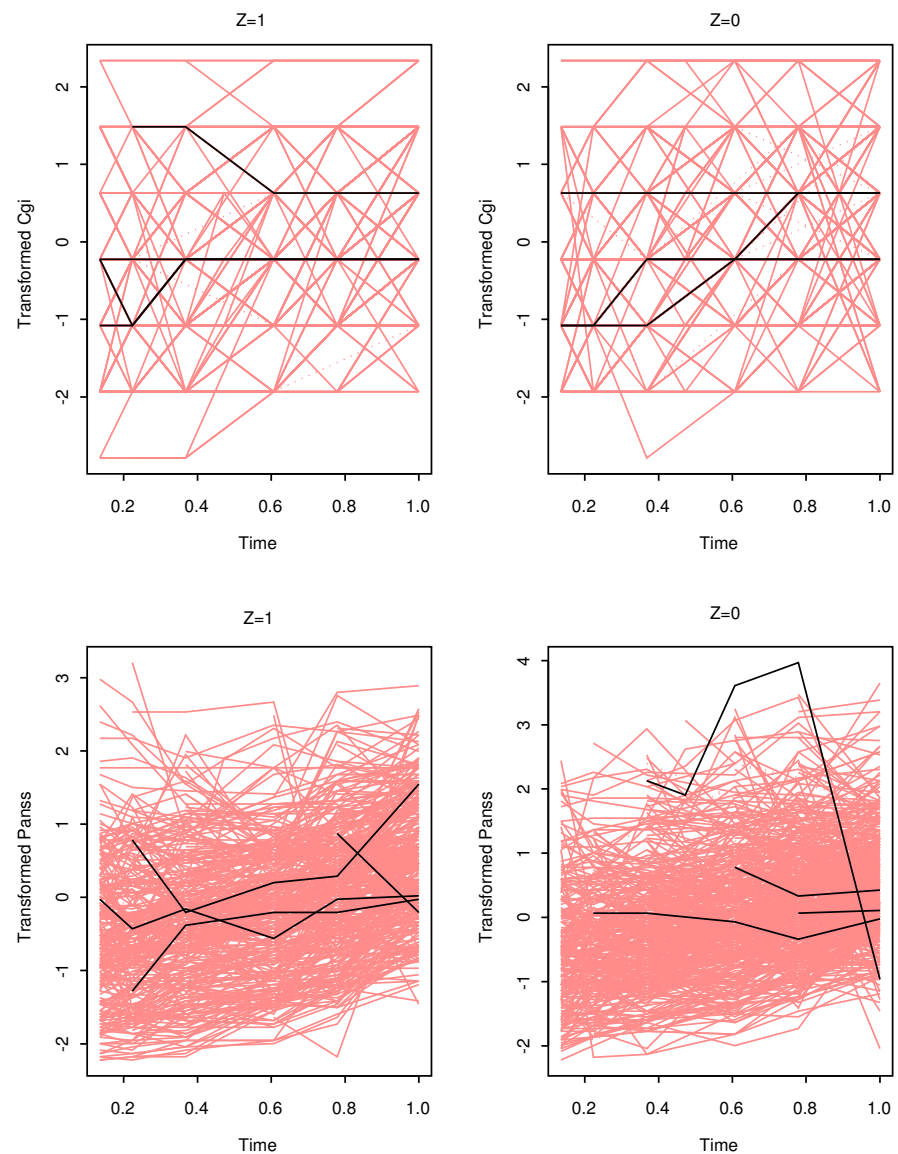

Figure 1: Psychiatric Study. Individual and mean profiles for each scale by treatment group. 